\title{
Autophagy, the Trojan horse to combat glioblastomas
}

\author{
Florence Lefranc, M.D., Ph.D., And Robert Kiss, Ph.D. \\ Department of Neurosurgery, Erasme University Hospital; and Laboratory of Toxicology, \\ Institute of Pharmacy, Free University of Brussels, Belgium
}

\begin{abstract}
$\checkmark$ Malignant gliomas, among which glioblastomas constitute the largest group, are characterized by a dramatically diffuse infiltration into the brain parenchyma with, as a consequence, the fact that no patient with glioblastoma multiforme (GBM) has been cured to date. Migrating GBM cells are resistant to apoptosis (Type I programmed cell death), and thus to radiotherapy and conventional chemotherapy, because of the constitutive activation of several intracellular signaling pathways, of which the most important identified to date are the pathways controlled by phosphatidylinositol 3-kinase, Akt, and the mammalian target of rapamycin (mTOR). Migrating GBM cells seem to be less prone to resist autophagy (Type II programmed cell death), and disruption of the pathway controlled by mTOR induces marked autophagic processes in GBM cells. Temozolomide is the most efficacious cytotoxic drug employed today to combat glioblastoma, and this drug exerts its cytotoxic activity through proautophagic processes. Thus, autophagy represents a kind of Trojan horse that can be used to bypass, at least partly, the dramatic resistance of glioblastoma to radiotherapy and proapoptotic-related chemotherapy.
\end{abstract}

KEY WORDS • autophagy • glioblastoma multiforme • migration • temozolomide

$\mathrm{G}$ LIOMAS account for more than $50 \%$ of all brain tumors and are by far the most common primary brain tumors in adults. ${ }^{19,23}$ They include three histopathological subgroups characterized by different levels of aggressiveness and malignancy: ependymomas $(<10 \%$ of all gliomas), oligodendrogliomas (10-30\%), and astrocytomas $(60-70 \%)$. All glial tumors are malignant, except for the following: benign ependymomas, subependymomas, gangliocytomas, dysembryoplastic neuroectodermal tumors, subependymal giant-cell astrocytomas, and nodular pilocytic astrocytomas, which taken together account for less than $10 \%$ of glial tumors. Malignant astrocytic gliomas are associated with dismal prognoses because of their ability to infiltrate diffusely into the normal brain

Abbreviations used in this paper: $\mathrm{EGFR}=$ epidermal growth factor receptor; $\mathrm{GBM}=$ glioblastoma multiforme; $\mathrm{MGMT}=\mathrm{O}^{6}$ methylguanine-DNA methyltransferase; $\mathrm{mTOR}=$ mammalian target of rapamycin; NF- $\mathrm{B}=$ nuclear factor $-\kappa \mathrm{B} ; \mathrm{PI} 3 \mathrm{~K}=$ phosphatidylinositol 3-kinase. parenchyma. These so-called "diffuse" astrocytic gliomas include tumors categorized by the World Health Organization as Grades II, III, and IV. Glioblastomas multiforme (Grade IV) account for approximately $50 \%$ of all glial tumor types and are associated with the worst prognoses. Patients with these tumors have an average life expectancy of 1 year with the standard treatment of resection followed by radiotherapy. ${ }^{23,34}$ However, an international clinical trial conducted by Stupp, et al.,${ }^{34}$ has recently revealed that the addition of the chemotherapy agent temozolomide to radiation therapy increases survival in patients with glioblastoma. A companion laboratory study published by Hegi, et al. ${ }^{11}$ has offered hope of even greater improvements in survival in the future, through identification of a molecular change in the tumor that allows the prediction of benefit from the new treatment. The combined work published by Stupp, et al., and Hegi, et al., is therefore being seen as a significant breakthrough in medical research for patients with glioblastoma. These clinical data strongly suggest that delivering temozolomide to patients with glioblastoma as soon as radiotherapy begins, instead of 
administering temozolomide after radiotherapy once the glioblastoma recurs, has a significant impact on patient survival. Indeed, the 2-year survival rate of patients with glioblastoma who benefitted from conventional treatment is lower than $10 \%$, whereas it exceeds $20 \%$ in the series of patients treated by Stupp and colleagues. ${ }^{34}$ Temozolomide is a proautophagic drug, not a proapoptotic one. ${ }^{17}$ Glioblastomas naturally resist apoptosis..$^{9,12,23}$ We thus review the following in the present paper: 1) the major signaling pathways that make glioblastomas resistant to apoptosis and, in parallel, the potential association of antimigratory compounds with proapoptotic cytotoxic drugs; 2) the signaling pathways distinctly activated by proautophagic drugs compared with proapoptotic ones; 3 ) the major scientific data already obtained by researchers to prove that temozolomide is actually a proautophagic drug that overcomes glioblastoma cell resistance to apoptosis; and 4) the most convincing clinical data obtained to date with respect to temozolomide in the treatment of patients with glioblastoma.

\section{Resistance of GBMs to Therapy}

Part of the switch between proliferation and migration processes is controlled by the CAS/Crk pathway. ${ }^{20}$ This pathway is also involved in the suppression of apoptosis during cell migration. ${ }^{5}$ Kapoor and O'Rourke ${ }^{18}$ recently reviewed the mechanism of the receptor tyrosine kinase signaling involved in gliomagenesis, with particular emphasis on the cooperative interaction between the signaling and the cell cycle-regulatory machinery. Kapoor and O'Rourke and our group ${ }^{23}$ have recently reviewed those growth factor inhibitors (including EGFR and plateletderived growth factor receptor inhibitors) that might contribute therapeutic benefits in adjuvant chemotherapies aimed to combat and destroy isolated proliferating GBM cells that escaped surgery and radiotherapy.

The natural resistance of glioblastomas to radiotherapy and chemotherapy is contributed, at least according to current scientific knowledge, by the PTEN/Akt/PI3K/mTOR/ NF- $\mathrm{B}$ pathway, as shown in Fig. 1. The complexity of cell survival through Akt signaling was recently reviewed by McCormick, ${ }^{25}$ and the mTOR pathway as a target for cancer therapy has been reviewed by Bjornsti and Houghton. ${ }^{3}$

The activity of the PI3K/Akt pathway is often up-regulated in brain tumors due to excessive stimulation by growth factor receptors and Ras. ${ }^{28,30}$ In fact, GBMs frequently carry mutations in the PTEN tumor suppressor gene, whose properties are closely related to its inhibitory effect on the PI3K-dependent activation of Akt signaling..$^{21,28}$ The activation of the PI3K pathway is significantly associated with higher tumor grade, lower levels of apoptosis, and an adverse clinical outcome in the case of human gliomas. ${ }^{4}$ Narita, et al.,${ }^{27}$ and Choe, et al.,${ }^{6}$ suggest that the PI3K/Akt pathway is a target of particular interest in the case of GBMs with aberrant EGFR expression because the aberrant EGFR expression and abnormal PI3K/ Akt signaling also modulate the migration levels of tumor astrocytes. ${ }^{23}$ Authors of a number of publications have already reported that an aberrantly activated PI3K/Akt pathway renders tumor cells resistant to cytotoxic insults, in- cluding those related to anticancer drugs. ${ }^{14,33}$ Shingu, et al., ${ }^{32,33}$ have shown that the inhibition of this pathway augments or even restores the effectiveness of chemotherapy on glioma cells. Also, PI3K inhibitors could be used to reduce the levels of tumor astrocyte migration, a feature that could restore a certain level of apoptosis in these cells. ${ }^{14}$

Cell survival through Akt signaling also involves the $\mathrm{NF}-\kappa \mathrm{B}$ pathway because Akt signals to various cell-death regulators, including inhibitor $\mathrm{\kappa B}$ kinase, which controls NF- $\kappa \mathrm{B}$ activity. ${ }^{15}$ Such NF- $\kappa \mathrm{B}$ activity plays a dramatic role in gliomagenesis. Indeed, the NF- $\mathrm{BB}$ signaling pathway is constitutively activated in a large proportion of glioblastomas, ${ }^{26}$ which enables glioblastomas (and also other cancer cell types) to resist cytotoxic insults. ${ }^{1,2}$ The constitutive activation of Akt and NF- $\kappa \mathrm{B}$ contributes significantly to the progression of diffuse gliomas, and the activation of Akt may lead to NF- $\kappa$ B activation in highgrade gliomas. ${ }^{37}$

Rapamycin inhibits the phosphorylation of the retinoblastoma protein, and rapamycin-treated cells are therefore not fully committed to enter the S-phase after their release from drug-induced $\mathrm{G}_{1}$ arrest. ${ }^{31}$ Constitutive retinoblastoma phosphorylation frequently occurs in GBMs due to mutation-induced p16 gene inactivation, ${ }^{19}$ and mTOR can also control cell migration in GBMs. ${ }^{23}$

\section{Patterns of Cell Death}

Cell death can be categorized as apoptotic and nonapoptotic. ${ }^{29}$ The term apoptosis often has been used interchangeably with the term "programmed cell death." Apoptosis is the principal mechanism by which cells are physiologically eliminated. The original definition of apoptosis as a form of cell death distinct from necrosis was based on morphological criteria (Table 1). Necrosis, traditionally considered an unregulated, passive, energyindependent form of cell death, has been identified as another form of programmed cell death. Extensive failure of normal physiological pathways that are essential for maintaining cellular homeostasis, such as regulation of ion transport, energy production, and $\mathrm{pH}$ balance, can lead to necrosis. A classic example of a necrotic condition is ischemia that leads to a drastic depletion of oxygen, glucose, and other trophic factors and evokes massive necrotic death of endothelial cells and cells of surrounding tissues. This may occur in the center of large malignant tumors and is characteristic of GBMs. ${ }^{19}$ Because GBM cells carry mutations that inactivate apoptotic pathways, ${ }^{18,23}$ necrosis could also represent an alternative pathway for tumor cells to be eliminated. In this paper, we describe autophagy, a mechanism for nonapoptotic programmed cell death that is distinct from apoptosis and necrosis, according to criteria of morphology, biochemistry, and the mechanisms of molecular pathways (Table 1). ${ }^{13,29}$

\section{Autophagy: Potential Trojan Horse for Glioblastomas}

Whereas apoptosis is a caspase-dependent process characterized by the condensation of cytoplasm and the preservation of organelles, essentially without any autophagic degradation, autophagic cell death is a caspase-independent process that exhibits an extensive autophagic degra- 
TABLE 1

Comparison of apoptosis, autophagy, and necrosis*

\begin{tabular}{lccc}
\hline \hline \multicolumn{1}{c}{ Characteristic } & $\begin{array}{c}\text { Apoptosis } \\
\text { (Type I PCD) }\end{array}$ & $\begin{array}{c}\text { Autophagy } \\
\text { (Type II PCD) }\end{array}$ & Necrosis \\
\hline $\begin{array}{l}\text { morphological change } \\
\text { nucleus fragmentation }\end{array}$ & + & - & - \\
chromatin condensation & + & - & - \\
apoptotic body formation & + & - & - \\
cytoplasmic vacuolation & - & + & + \\
organelle degradation & - & + & + \\
mitochondrial swelling & sometimes & late & + \\
cytoplasmic swelling and & & & \\
membrane breakdown & - & - & + \\
biochemical feature & & & - \\
caspase activity (caspase 3) & + & - & - \\
PARP & cleavage & - & activation \\
lysosomal activity (cathepsin B) & - & + & - \\
molecular pathway & & & \\
DAP & + & + & + \\
PI3K, mTOR & - & + & \\
Bcl-2 proteins \& cytochrome c & + & - & \\
\hline
\end{tabular}

$*$ DAP $=$ death-associated proteins; PARP $=$ poly (ADP-ribose $)$ polymerase PCD $=$ programmed cell death $;+=$ present $;-=$ absent.

dation of the Golgi apparatus, the polyribosomes, and the endoplasmic reticulum, with all these features preceding the destruction of the nucleus (Table 1). ${ }^{13,22,29}$ In fact, autophagy was originally defined as a process of protein recycling. ${ }^{17,22}$ Autophagy begins with the sequestering of cytoplasmic organelles in membrane vacuoles called autophagosomes, which then fuse with lysosomes, where the materials inside are degraded and recycled..$^{17,22}$ Figure 2 details the pathways that are activated during proautophagic processes.

In some cases, apoptotic and autophagic cell death coincide in vivo in certain tissues, and in rare cases, both morphologies may coincide within the same cells. ${ }^{29}$ Both apoptosis and autophagy are controlled by death-associated proteins. Mariani, et al., ${ }^{24}$ have shown that migrating glioma cells overexpress death-associated protein-3, with this overexpression conferring an increased resistance to camptothecin-induced apoptosis; this resistance can be overcome by effective death-associated protein-3 antisense treatment. ${ }^{24}$

Because mTOR seems to be involved in Type II autophagic programmed cell death, ${ }^{7}$ inactivation of mTOR also can induce autophagy. Indeed, mTOR is regulated by mitochondrial dysfunction and the depletion of adenosine triphosphate levels, which are effects inducible by, for example, modifications to cyclic adenosine monophosphate levels or osmotic stress. As illustrated in Figs. 1 and 2, mTOR is a downstream effector of the PI3K/Akt signaling pathway, and it is also a central modulator of cell proliferation in malignant gliomas. ${ }^{36}$ In fact, rapid tumor proliferation, which can result from low apoptotic levels, may contribute to the clinical radioresistance of GBMs, and the disruption of mTOR signaling by rapamycin returns radioresistant GBM cells to certain levels of radiosensitivity. ${ }^{8}$ Takeuchi, et al. ${ }^{35}$ showed that rapamycin induced autophagy but not apoptosis in rapamycin-sensitive malignant glioma U87-MG and T98G cells by inhibiting the function of mTOR. In contrast, in rapamycin-resistant

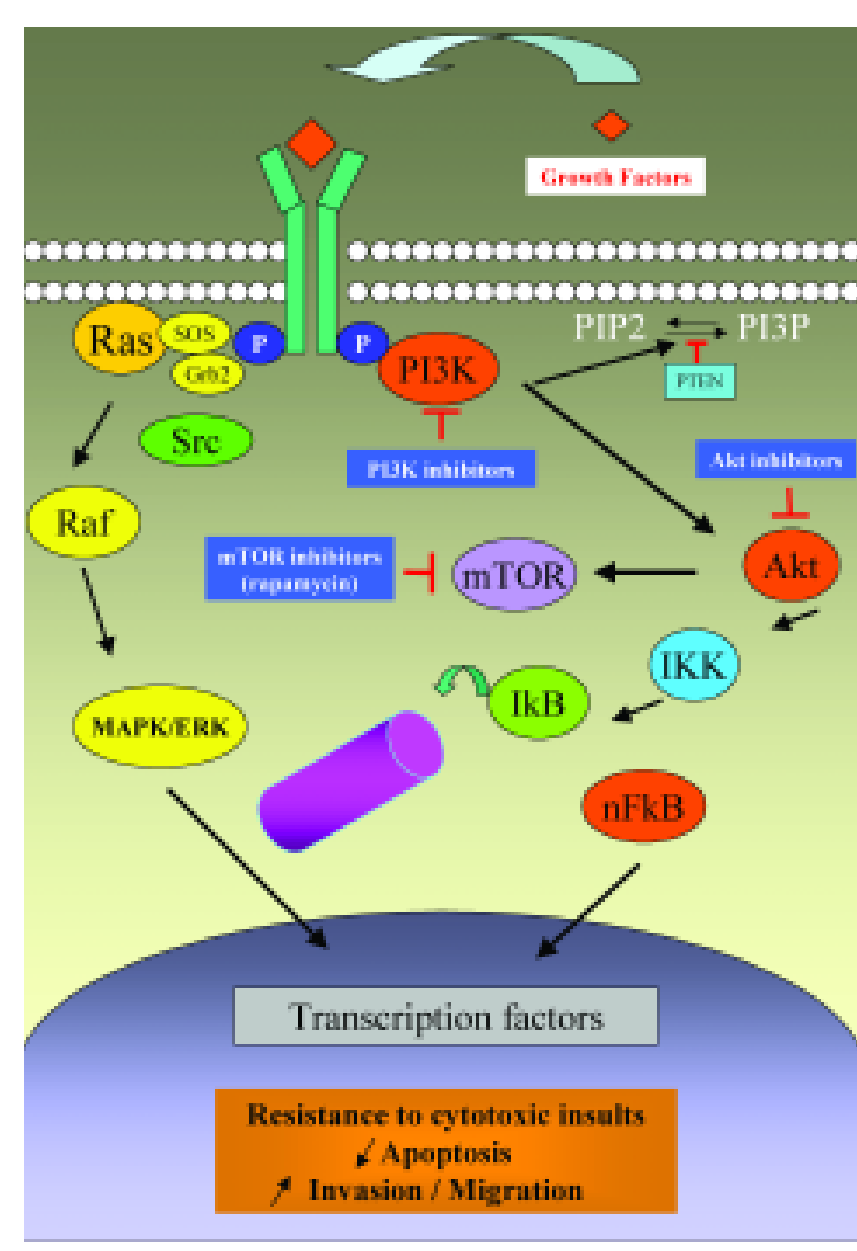

FIG. 1. Diagram showing pathways involved in the natural resistance of glioblastomas to radiotherapy and chemotherapy. Pathways involved in cytotoxic insult resistance are shaded orange and the inhibitors that could be used to restore the chemosensitivity to proapoptotic drugs are shaded blue. ERK = extracellular signal-regulated kinase; IKK = inhibitor $\mathrm{\kappa B}$ kinase; MAPK = mitogen-activated protein kinase; PIP2 = phosphatidylinositol 4,5biphosphate; PI3P = phosphatidylinositol 3-phosphate.

U373-MG cells, the inhibitory effect of rapamycin was minor. ${ }^{35}$ A PI3K inhibitor (LY294002) and an Akt inhibitor (7-hydroxystaurospaurine) both synergistically sensitized U87-MG and T98G as well as U373-MG cells to rapamycin by stimulating the induction of autophagy. ${ }^{36}$ Enforced expression of active Akt in GBM cells suppressed the combined effects of LY294002 or 7-hydroxystaurospaurine, whereas dominant-negative Akt expression was sufficient to increase the sensitivity of GBM cells to rapamycin. ${ }^{36}$ Based on these data, Takeuchi and colleagues ${ }^{35}$ concluded that rapamycin exerts its antitumor effect on GBM cells by inducing autophagy, and they suggest that in GBM cells a disruption of the PI3K/Akt signaling pathway could greatly enhance the effectiveness of mTOR inhibitors, as has already been demonstrated for proapoptotic drugs. ${ }^{23}$ Compounds that induce autophagy are also active against multidrug-resistant cells. ${ }^{10}$

Autophagy could thus represent a new approach to be investigated in relation to both cell proliferation and mi- 


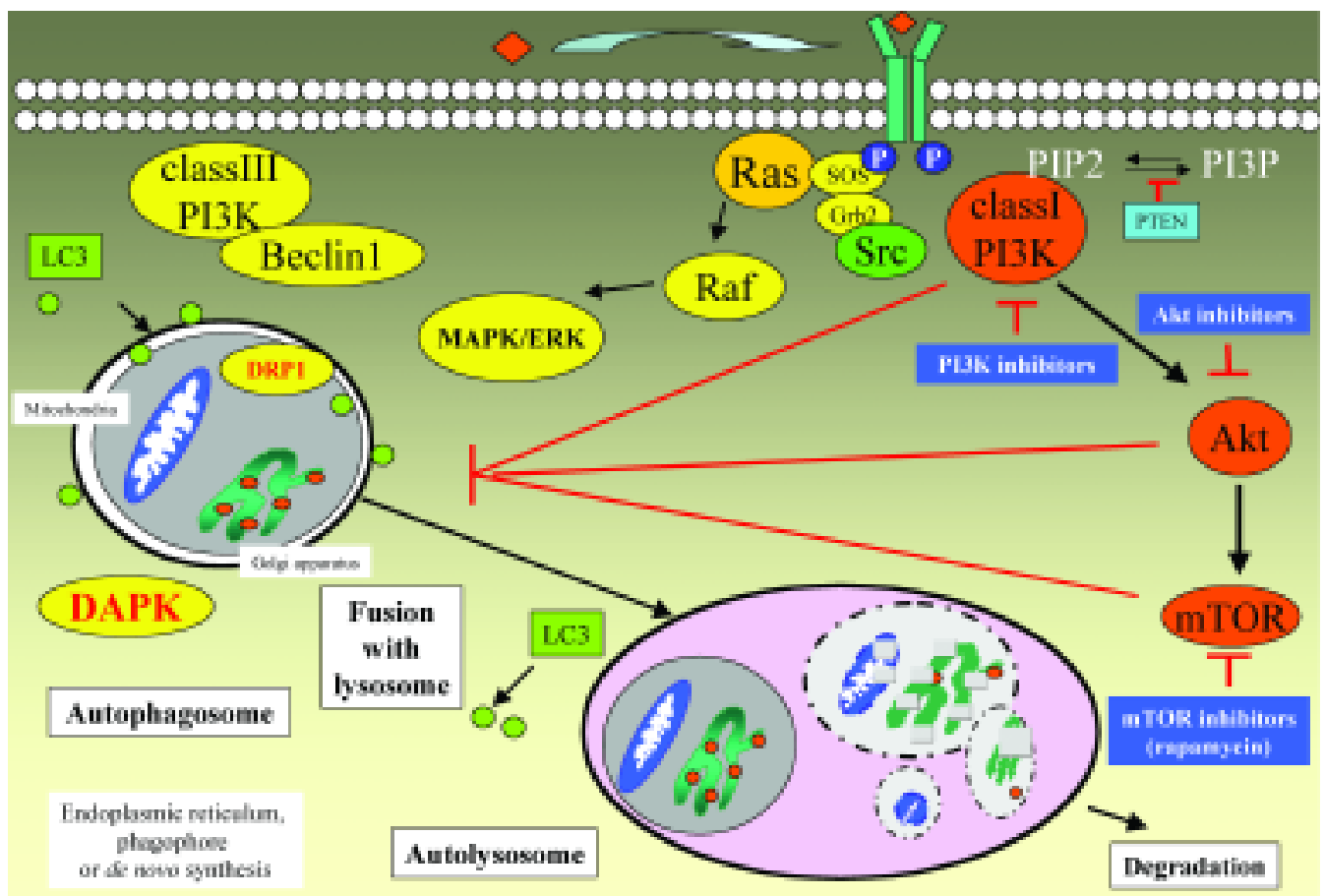

FIG. 2. Diagram showing the molecular regulation of autophagy. In the presence of growth factors, growth factor receptor signaling activates Class I PI3K, which activates the downstream targets Akt and mTOR, resulting in inhibition of autophagy. Rapamycin, an inhibitor of mTOR, induces autophagy. The PTEN gene mutation promotes Akt activation. Ras has a dual effect on autophagy; when it activates Class I PI3K, autophagy is inhibited, but when it activates the Raf/mitogen-activated protein kinase/extracellular signal-regulated kinase cascade, autophagy is stimulated. A complex of Class III PI3K and beclinl (a tumor suppressor gene) is required for a proximal step in autophagy. Autophagy is also induced by the cell death-associated protein kinase (DAPK) and the death-associated related protein kinase 1 (DRP1). Class III PI3K has been shown to be required for both autophagic vesicle formation and vesicular transport to the lysosome. LC $3=$ microtubule-associated protein light-chain 3.

gration features in malignant gliomas, and inhibition of mTOR activity therefore represents a possible method to combat apoptotic-resistant migrating glioblastoma cells. Clinical trials with mTOR inhibitors are proceeding on patients with recurrent malignant gliomas. ${ }^{28}$ Temozolomide, the proautophagic drug that is associated with the most promising clinical efficacy, is discussed in the following section.

\section{Therapeutic Benefits of Temozolomide}

The cytotoxicity of temozolomide is thought to be mainly due to the formation of $\mathrm{O}^{6}$-methylguanine in DNA, which mispairs with thymine during the next cycle of DNA replication. ${ }^{17}$ Glioma cells thus respond to temozolomide by undergoing $\mathrm{G}_{2} / \mathrm{M}$ arrest but will ultimately die from autophagy. Researchers at the M. D. Anderson Cancer Center ${ }^{16,17,35}$ elegantly demonstrated that temozolomide induces autophagic, but not apoptotic, processes in human glioblastoma cells. Knowing that $\mathrm{O}^{6}-\mathrm{MGMT}$ is a DNA repair enzyme that limits the efficacy of temozolomide in glioblastoma cells, Kanzawa, et al., ${ }^{16}$ first showed that $\mathrm{O}^{6}$-benzylguanine, an MGMT inhibitor, can render previously resistant glioblastoma cells sensitive to temozolomide. These authors observed that the mechanism of cell demise following treatment with $\mathrm{O}^{6}$-benzylguanine and temozolomide seemed to be autophagy and not apoptosis. Kanzawa, et al., ${ }^{17}$ then showed that at a clinically achievable dose $(100 \mu \mathrm{M})$, temozolomide induced autophagy, but not apoptosis, in malignant glioma cells. After the treatment with temozolomide, microtubule-associated protein light-chain 3, a mammalian homolog of Apg8p/ aut7p, which is essential for amino acid starvation-induced autophagy in yeast, was recruited on autophagosome membranes. When autophagy was prevented at an early stage by 3 -methyladenine, a PI3K kinase inhibitor (Figs. 1 and 2), not only the characteristic pattern of lightchain 3 localization, but also the antitumor effect of temozolomide was suppressed.

Temozolomide is less toxic to the hematopoietic progenitor cells than conventional chemotherapeutic agents because it does not result in chemical cross-linking of the DNA strands, whereas other alkylating agents do crosslink the DNA. ${ }^{17}$ Thus, Stupp, et al. ${ }^{34}$ sought to determine whether standard treatment can be extended by adding temozolomide to radiotherapy, both concomitantly and as an adjuvant treatment, without deleterious impact on quality of life for patients. A total of 573 patients from 85 centers who had newly diagnosed, histologically confirmed glioblastoma were randomly assigned to receive radiotherapy alone or radiotherapy plus temozolomide. The treatment options were as follows: 1) radiotherapy alone, consisting of focal irradiation in daily fractions of 2 Gy given 5 days per week 
for 6 weeks, to a total of $60 \mathrm{~Gy}$; or 2) radiotherapy plus continuous daily temozolomide, with radiotherapy consisting of $75 \mathrm{mg} / \mathrm{m}^{2}$ body surface area per day, 7 days per week from the first to the last day of radiotherapy, followed by six cycles of adjuvant temozolomide at a dose of 150 to $200 \mathrm{mg} /$ $\mathrm{m}^{2}$ for 5 days during each 28-day cycle.

The clinical data obtained by Stupp and colleagues ${ }^{34}$ showed a $26 \%$ survival rate at 2 years in those patients treated with the new combined therapy, compared with only $10 \%$ in those receiving radiotherapy alone. A companion laboratory study led by Hegi, et al.,"11 offers the promise of even greater impact in the future for the combined modality treatment proposed by Stupp and colleagues. ${ }^{34}$ Indeed, the data obtained by Hegi and colleagues ${ }^{11}$ showed that patients who had glioblastoma that contained a methylated $\mathrm{O}^{6}$-MGMT promoter benefited from temozolomide, whereas those who did not showed less benefit.

\section{Conclusions}

Glioblastomas are associated with dismal prognoses because they diffusely infiltrate the brain parenchyma, therefore rendering total resection of all tumor cells impossible. In addition, migrating glioblastoma cells, which can escape the most sophisticated surgical approaches, are resistant to apoptosis and thus to radiotherapy and most of the chemotherapeutic agents that are associated with proapoptotic effects, including procarbazine, vincristine, lomustine, and carmustine. Autophagy is another type of cell death against which glioblastoma cells are less resistant. Temozolomide, a proautophagic cytotoxic drug, contributes actual therapeutic benefits in patients with glioblastoma who already can benefit from surgery and radiotherapy. The therapeutic benefits of temozolomide can be reinforced by mTOR inhibitors, whose therapeutic benefits can in turn be reinforced by PI3K and Akt inhibitors. Such PI3K and Akt inhibitors are also able to restore a certain level of sensitivity in migrating apoptosis-resistant glioblastoma cells. Thus, novel successes in the fight against glioblastoma could be achieved by combining mTOR, PI3K, and Akt inhibitors with temozolomide as adjuvant chemotherapies, in which EGFR and platelet-derived growth factor receptor inhibitors are also hopeful agents to combat the proliferation processes of glioblastoma cells.

\section{References}

1. Aggarwal BB: Nuclear factor-кB: the enemy within. Cancer Cell 6:203-208, 2004

2. Baldwin AS: Control of oncogenesis and cancer therapy resistance by the transcription factor NF-кB. J Clin Invest 107: 241-246, 2001

3. Bjornsti MA, Houghton PJ: The TOR pathway: a target for cancer therapy. Nat Rev Cancer 4:335-348, 2004

4. Chakravarti A, Zhai G, Suzuki Y, et al: The prognostic significance of phosphatidylinositol 3-kinase pathway activation in human gliomas. J Clin Oncol 22:1926-1933, 2004

5. Cho SY, Klemke RL: Extracellular-regulated kinase activation and CAS/Crk coupling regulate cell migration and suppress apoptosis during invasion of the extracellular matrix. J Cell Biol 149:223-236, 2000

6. Choe G, Horvath S, Cloughesy TF, et al: Analysis of the phosphatidylinositol 3'-kinase signalling pathway in glioblastoma patients in vivo. Cancer Res 63:2742-2746, 2003
7. Desai BN, Myers BR, Schreiber SL: FKB12-rapamycin-associated protein associates with mitochondria and senses osmotic stress via mitochondrial dysfunction. Proc Natl Acad Sci U S A 99:4319-4324, 2002

8. Eshleman JS, Carlson BL, Mladek AC, et al: Inhibition of the mammalian target of rapamycin sensitizes U87 xenografts to fractionated radiation therapy. Cancer Res 62:7291-7297, 2002

9. Giese A, Bjerkvig R, Berens ME, et al: Cost of migration: invasion of malignant gliomas and implications for treatment. J Clin Oncol 21:1624-1636, 2003

10. Guo B, Hembruff SL, Villeneuve DJ, et al: Potent killing of paclitaxel-and doxorubicin-resistant breast cancer cells by calphostin $\mathrm{C}$ accompanied by cytoplasmic vacuolization. Breast Cancer Res Treat 82:125-141, 2003

11. Hegi ME, Diserens AC, Gorlia T, et al: $M G M T$ gene silencing and benefit from temozolomide in glioblastoma. N Engl J Med 352:997-1003, 2005

12. Hoelzinger DB, Mariani L, Weis J, et al: Gene expression profile of glioblastoma multiforme invasive phenotype points to new therapeutic targets. Neoplasia 7:7-16, 2005

13. Inbal B, Bialik S, Sabanay I, et al: DAP kinase and DRP-1 mediate membrane blebbing and the formation of autophagic vesicles during programmed cell death. J Cell Biol 157:455-468, 2002

14. Joy AM, Beaudry CE, Tran NL, et al: Migrating glioma cells activate the PI3-k pathway and display decreased susceptibility to apoptosis. J Cell Sci 116:4409-4417, 2003

15. Kain KH, Gooch S, Klemke RL: Cytoplasmic c-Abl provides a molecular 'rheostat' controlling carcinoma cell survival and invasion. Oncogene 22:6071-6080, 2003

16. Kanzawa T, Bedwell J, Kondo Y, et al: Inhibition of DNA repair for sensitizing resistant glioma cells to temozolomide. J Neurosurg 99:1047-1052, 2003

17. Kanzawa T, Germano IM, Komata T, et al: Role of autophagy in temozolomide-induced cytotoxicity for malignant glioma cells. Cell Death Differ 11:448-457, 2004

18. Kapoor GS, O'Rourke DM: Mitogenic signaling cascades in glial tumors. Neurosurgery 52:1425-1435, 2003

19. Kleihues P, Cavenee WK: Pathology and Genetics of Tumours of the Nervous System. Lyon: IARC Press, 2000

20. Klemke RL, Leng J, Molander R, et al: CAS/Crk coupling serves as a "molecular switch" for induction of cell migration. J Cell Biol 140:961-972, 1998

21. Knobbe CB, Reifenberger G: Genetic alterations and aberrant expression of genes related to the phosphatidyl-inositol-3' kinase/protein kinase B (Akt) signal transduction pathway in glioblastomas. Brain Pathol 13:507-518, 2003

22. Kondo Y, Kanzawa T, Sawaya R, et al: The role of autophagy in cancer development and response to therapy. Nat Rev Cancer 5:726-734, 2005

23. Lefranc F, Brotchi J, Kiss R: Possible future issues in the treatment of glioblastomas: special emphasis on cell migration and the resistance of migrating glioblastoma cells to apoptosis. J Clin Oncol 23:2411-2422, 2005

24. Mariani L, Beaudry C, McDonough WS, et al: Death-associated protein 3 (Dap-3) is overexpressed in invasive glioblastoma cells in vivo and in glioma cell lines with induced motility phenotype in vitro. Clin Cancer Res 7:2480-2489, 2001

25. McCormick F: Cancer: survival pathways meet their end. Nature 428:267-269, 2004

26. Nagai S, Washiyama K, Kurimoto M, et al: Aberrant nuclear factor- $\kappa \mathrm{B}$ and its participation in the growth of human malignant astrocytoma. J Neurosurg 96:909-917, 2002

27. Narita $Y$, Nagane M, Mishima K, et al: Mutant epidermal growth factor receptor signalling down-regulates p27 through activation of the phosphatidylinositol 3-kinase/Akt pathway in glioblastomas. Cancer Res 62:6764-6769, 2002

28. Newton HB: Molecular neuro-oncology and development of targeted therapeutic strategies for brain tumors. Part 2: PI3K/ Akt/PTEN, mTOR, SHH/PTCH and angiogenesis. Expert Rev Anticancer Ther 4:105-128, 2004 


\section{F. Lefranc and R. Kiss}

29. Okada H, Mak TW: Pathways of apoptotic and non-apoptotic death in tumor cells. Nat Rev Cancer 4:592-603, 2004

30. O'Rourke DM: Targeted molecular therapy in glial tumors. Neurosurgery 54:N9, 2004

31. Sekulić A, Hudson CC, Homme JL, et al: A direct linkage between the phosphoinositide 3-kinase-AKT signaling pathway and the mammalian target of rapamycin in mitogen-stimulated and transformed cells. Cancer Res 60:3504-3513, 2000

32. Shingu T, Yamada K, Hara N, et al: Growth inhibition of human malignant glioma cells induced by the PI3-k-specific inhibitor. J Neurosurg 98:154-161, 2003

33. Shingu T, Yamada K, Hara N, et al: Synergistic augmentation of antimicrotubule agent-induced cytotoxicity by a phosphoinositide 3-kinase inhibitor in human malignant glioma cells. Cancer Res 63:4044-4047, 2003

34. Stupp R, Mason WP, van den Bent M, et al: Radiotherapy plus concomitant and adjuvant temozolomide for glioblastoma. $\mathbf{N}$ Eng J Med 352:987-996, 2005

35. Takeuchi H, Kanzawa T, Kondo Y, et al: Inhibition of platelet- derived growth factor signalling induces autophagy in malignant glioma cells. Br J Cancer 90:1069-1075, 2004

36. Takeuchi H, Kondo Y, Fujiwara K, et al: Synergistic augmentation of rapamycin-induced autophagy in malignant glioma cells by phosphatidylinositol 3-kinase/protein kinase B inhibitors. Cancer Res 65:3336-3346, 2005

37. Wang H, Wang H, Zhang W, et al: Analysis of the activation status of Akt, NFKB, and Stat3 in human diffuse gliomas. Lab Invest 84:941-951, 2004

Manuscript received January 10, 2006.

Accepted in final form March 16, 2006.

Address reprint requests to: Florence Lefranc, M.D., Ph.D., Department of Neurosurgery, Erasme University Hospital-Université Libre de Bruxelles, Route de Lennik, 808-1070 Brussels, Belgium. email: fllefran@ulb.ac.be. 